\title{
AAVALIAÇÃO FORENSE DE CASOS DE VIOLÊNCIA DOMÉSTICA DURANTE O INICIO DO ISOLAMENTO PELA PANDEMIA COVID-19
}

\author{
THE FORENSICS ASSESSMENT OF DOMESTIC'S VIOLENCE CASES DURING THE START OF ISOLATION DUE TO \\ THE COVID-19 PANDEMIC
}

Os autores informam não haver conflito de interesse

ARTIGO ORIGINAL recebido 14/9/2020, aceito em 30/9/2020

Como citar: Rios AMFM et al. A avaliação forense de casos de violência doméstica durante o início do isolamento pela pandemia covid-19. Persp Med Legal Pericias Med. 2020; 5(3) https://dx.doi.org/10.47005/050302

Angelita Maria Ferreira Machado Rios (1 e 2) Currículo Lattes: 9216524646561227 - ID ORCID: 0000-0001-8363-4532 Alexia Oro dos Santos (2) Currículo Lattes: 1441450866216280 - ID ORCID: 0000-0001-7522-0102 Larissa de Oliveira Silveira ${ }^{(2)}$ Currículo Lattes: 0677168195955898 - ID ORCID: 0000-0002-3688-1773 Laura Chies Kercher (2) Currículo Lattes: 4939477268529245 - ID ORCID: 0000-0001-9818-9451 Livia Capuano Fogaça (2) ID ORCID: 0000-0002-6781-8347 Letiane Montagner Ifarraguirre (2) ID ORCID: 0000-0002-6628-3395

Eduarda Pasini Dein (2) Currículo Lattes: 3364513894095527 - ID ORCID: 0000-0002-1661-7094 Martha Rocha (1)

ID ORCID 0000-0002-6811-1669 Vanessa Machado Rios (3)

Currículo Lattes: 6033620740956681 - ID ORCID: 0000-0002-2123-3677

(1) Departamento Médico-Legal de Porto Alegre/Instituto Geral de Perícias-RS/ Porto Alegre/Brasil (autor principal)

(2) Faculdade de Medicina - Disciplina de Medicina Legal - Universidade Luterana do Brasil-Canoas/RS/Brasil

(revisão bibliográfica e tradução)

(3) Faculdade de Medicina - Disciplina de Medicina Legal - Universidade Luterana do BrasilCanoas/RS/Brasil (coordenação)

(4) Departamento Médico-Legal de Porto Alegre/Instituto Geral de Perícias-RS/ Porto Alegre/Brasil (coleta de dados)

(5) Pontifícia Universidade Católica do Rio Grande do Sul - Faculdade de Medicina/Porto Alegre/Brasil

(revisão de literatura e tradução)

e-mail: jonas.costella@gmail.com 


\section{RESUMO}

Introdução: o isolamento social é um recurso importante para diminuir a velocidade de contaminação durante a pandemia Covid-19. Entretanto, pode intensificar comportamentos abusivos entre casais ou membros da família.

Objetivo: apresentar dados demográficos e criminais de vítimas de violência doméstica avaliadas na Clínica Forense e Serviço Psicossocial do Departamento Médico-Legal de Porto Alegre.

Método: levantamento retrospectivo de dados, que analisou entrevistas de 47 pessoas vítimas de violência doméstica, entre abril e maio de 2020. A amostra foi constituída por 30 casos de violência por parceiro íntimo (VPI) e 17 casos de violência familiar (VF). Foram analisadas variáveis sociodemográficas e criminais e a opinião sobre a dinâmica da violência na quarentena.

Resultado: a violência doméstica atingiu predominantemente as vítimas do sexo feminino ( $96,6 \%$ da violência por parceiro íntimo e $58,8 \%$ da violência familiar). O sexo masculino foi vítima de violência familiar nas faixas etárias extremas. As mulheres sofreram violência ao longo do ciclo vital, mas agressões por parceiros íntimos apresentaram picos na adolescência e juventude. Crianças foram agredidas pelas figuras de proteção e vítimas com mais de 60 anos de idade sofreram violência física por parte de filhos ou genros. Na violência por parceiro íntimo, 53\% da amostra relatou agressão por ex-companheiros e o tempo de separação variou entre 15 dias e dois anos. Na opinião de $90,3 \%$ das mulheres, a quarentena não modificou a frequência ou intensidade dos atos abusivos.

Conclusão: a violência no ambiente doméstico atinge predominantemente as pessoas em situação de vulnerabilidade como crianças, mulheres e idosos. Não foi possível fazer inferência sobre o aumento de casos de violência durante o isolamento social.

Palavras-chave: violência de gênero, violência doméstica, quarentena Covid-19

\section{ABSTRACT}

Introduction: social isolation is an important resource to slow down the speed of contamination during the COVID-19 pandemic, however it can intensify abusive behavior between couples or family members.

Objective: to present demographic and criminal data of victims of domestic violence assessed at the Clinic of Forensics and Psychosocial Service of the Medical-Legal Department of Porto Alegre.

Method: retrospective survey of data, which analyzed interviews of 47 victims of domestic violence, between April and May 2020. The sample consisted of 30 cases of intimate partner violence (IPV) and 17 cases of family violence (VF). Sociodemographic and criminal variables and the opinion on the dynamics of violence in quarantine were analyzed.

Result: domestic violence predominantly affected female victims $(96.6 \%$ of intimate partner violence and $58.8 \%$ of family violence). The male gender was a victim of family violence in extreme age groups. Women suffered violence throughout their life cycle, but aggressions by intimate partners showed peaks in adolescence and youth. Children were abused by close relatives and victims over 60 years of age suffered physical violence from their children or sons-in-law. In intimate partner violence, $53 \%$ of the sample reported aggression by ex-partners, between 15 days and two years' timeframe. In the opinion of $90.3 \%$ of women, quarantine did not change the frequency or intensity of abusive acts.

Conclusion: violence in the domestic environment predominantly affects people in vulnerable situations such as children, women and the elderly. It was not possible to reach conclusions regarding increase in domestic violence during the quarantine.

Keywords: gender violence, domestic violence, quarantine Covid-19 


\section{INTRODUÇÃO}

Durante a pandemia Covid-19, o isolamento social é um recurso importante para diminuir a velocidade de contaminação, entretanto pode acarretar alterações econômicas, físicas e emocionais para algumas famílias. Neste cenário a violência doméstica pode ser desencadeada ou intensificada, evoluindo para formas graves como o feminicídio ou familicídio. $(1,2,3,4)$. Este comportamento abusivo pode ocorrer entre um casal (Violência por Parceiro Íntimo - VPI) ou em uma família (Violência Familiar - VF). Qualquer pessoa pode ser vítima de uma relação abusiva, mas as mulheres e crianças apresentam um risco maior de violência física ou sexual, enquanto idosos e pessoas com necessidades especiais são frequentemente negligenciadas (5).

Para muitos agressores domésticos, o isolamento da vítima é uma forma de controle e redução de oportunidades de revelação da violência (1). Normalmente, estas vítimas sofrem restrição no uso das redes sociais, internet e telefone celular, permanecendo sem o suporte emocional ofertado por amigos, família, escolas, igrejas e outras instituições durante a quarentena. $(6,7,8,9)$. Com o fechamento de restaurantes, bares e outros locais onde ocorre consumo de álcool, muitas vezes, os agressores passam a consumir na própria residência, aumentando os riscos para a violência doméstica. Durante a pandemia Covid-19, muitos países têm registrado o aumento de homicídios no ambiente doméstico $(1,10,11)$.

Ao analisar o impacto social de grandes desastres naturais (terremotos, furacões, tsunamis), estudos demonstraram que algumas taxas de crimes podem não sofrer modificações, mas as taxas de violência doméstica frequentemente sofrem elevação. $(8,9,10,11,12)$. Seguindo o modelo de outros eventos de grande impacto, espera-se que a violência doméstica se mantenha nas famílias disfuncionais durante a pandemia e muitos anos após. Estão sendo notificados aumentos significativos de casos na Argentina, Dinamarca, Espanha, Itália, Reino Unido e nos Estados Unidos. Na China, a notificação de casos triplicou e na França houve aumento de $30 \%$ dos relatos de violência doméstica. No Brasil, estima-se um aumento de 40 a $50 \%$ de casos $(11,13)$, com elevação de $17 \%$ no número das ligações com denúncias de violência contra mulheres durante o mês de março, período inicial do distanciamento social (14). Além das restrições financeiras e de movimentos, as mulheres acabam vivenciando o aumento do trabalho doméstico, dos cuidados com crianças, idosos e familiares doentes (15).

$\mathrm{Na}$ notificação de violência doméstica, principalmente na modalidade perpetrada por parceiro íntimo (VPI), a própria vítima costuma procurar auxílio das autoridades, aproveitando ocasiões em que o agressor não se encontra na residência. Entretanto, essas oportunidades para busca de ajuda diminuem durante o isolamento social (14). O mesmo não ocorre quando se trata da violência contra crianças (VF), onde a intervenção de vizinhos, amigos ou professores é relevante para a denúncia dos eventos abusivos (9). Estima-se que muitos casos de violência doméstica permaneçam sem notificação durante o período de isolamento social, evidenciando o risco da vítima em permanecer no domicílio com o abusador (5). Algumas vítimas também relataram receio de procurar atendimento médico por medo do contágio do Covid-19 (1).

A violência doméstica tem sido um problema social crescente no Brasil e as pesquisas nacionais relacionadas com o isolamento social na pandemia Covid-19 ainda são escassas. Desta forma, o estudo proposto tem por objetivo apresentar dados demográficos de vítimas deste tipo de violência, nas modalidades VPI ou VF, entre abril e maio de 2020 e que foram avaliadas na Clínica Forense e Serviço Psicossocial do Departamento Médico-Legal (DML) de Porto Alegre. Serão avaliadas variáveis que permitam estabelecer um breve perfil epidemiológico destas pessoas, contribuindo para inseri-las nas políticas sociais existentes.

\section{METODOLOGIA}

Este estudo transversal, com levantamento retrospectivo de dados, analisou entrevistas de 47 pessoas com história de violência doméstica, durante os meses de abril e maio de 2020, realizadas por um técnico em Serviço Social do Departamento Médico-Legal de Porto Alegre. Esta atividade de acolhimento psicossocial foi ofertada às pessoas que aguardavam a realização de perícias na Clínica Médica Forense, no horário 
compreendido entre $8 \mathrm{~h}$ e $18 \mathrm{~h}$, de segunda à sextafeira; e realizado o encaminhamento posterior de demandas para a rede de assistência pública e privada. A população de abrangência da Clínica Médico-legal em estudo é de 2.216 .975 habitantes, considerando a população da capital e mais oito cidades da região metropolitana. Entretanto, com o fechamento de alguns postos forenses nas cidades vizinhas, pessoas de outras cidades também procuram a capital para realizar exames médicolegais. O distanciamento social na cidade de Porto Alegre teve início no dia 17 de março de 2020.

As formas de violência doméstica foram classificadas em violência por parceiro íntimo (VPI) e violência familiar (VF). A amostra foi constituída por 30 casos de VPI e 17 casos de VF. As variáveis sociodemográficas analisadas neste estudo foram: sexo, cor da pele, idade e procedência das vítimas. Foram também pesquisadas variáveis de interesse forense: vitimização prévia, grau de relacionamento com (o) agressor(es), tipo de violência e instrumento utilizado no evento abusivo. Às mulheres vítimas de VPI, foi solicitada a opinião sobre o isolamento social (pandemia Covid-19) na dinâmica da violência e verificado se houve a solicitação de medida protetiva. As informações foram obtidas através do Protocolo Geral de Perícias do Instituto Geral de Perícias, notificações policiais e entrevistas com os periciados.

A pesquisa foi autorizada pelo Setor de Ensino e Pesquisa do Departamento Médico-Legal de Porto Alegre/Instituto Geral de Perícias-RS.

\section{RESULTADOS}

\section{Sexo}

Neste estudo, a violência doméstica atingiu predominantemente as vítimas do sexo feminino para ambas as modalidades abordadas. A violência entre parceiros (VPI) foi notificada por 29 mulheres (96,6\% da amostra) e apenas um homem (3,3\% da amostra). Nos casos de VF foram notificados casos envolvendo 10 mulheres ( $58,8 \%$ da amostra) e sete homens (41,1\% da amostra).

\section{Cor da pele}

Em nosso estudo foi observado que $78,7 \%$ da amostra (37 casos) apresentavam cor da pele branca. Ambas cores de pele preta (5 casos) e mista
(5 casos) corresponderam a $21,2 \%$ da amostra. Todos os homens (crianças e adultos) vítimas de violência possuíam a cor de pele branca.

\section{Faixa etária}

As faixas etárias das vítimas de violência no âmbito doméstico apresentaram distribuição distinta nas duas modalidades estudadas: violência por parceiro íntimo (VPI) e violência familiar (VF). O sexo masculino foi predominantemente vítima de violência familiar nas faixas etárias extremas: primeira infância e acima de sessenta anos (Figura 1). O único relato masculino de agressão por parceira ocorreu em um indivíduo com 51 anos de idade.

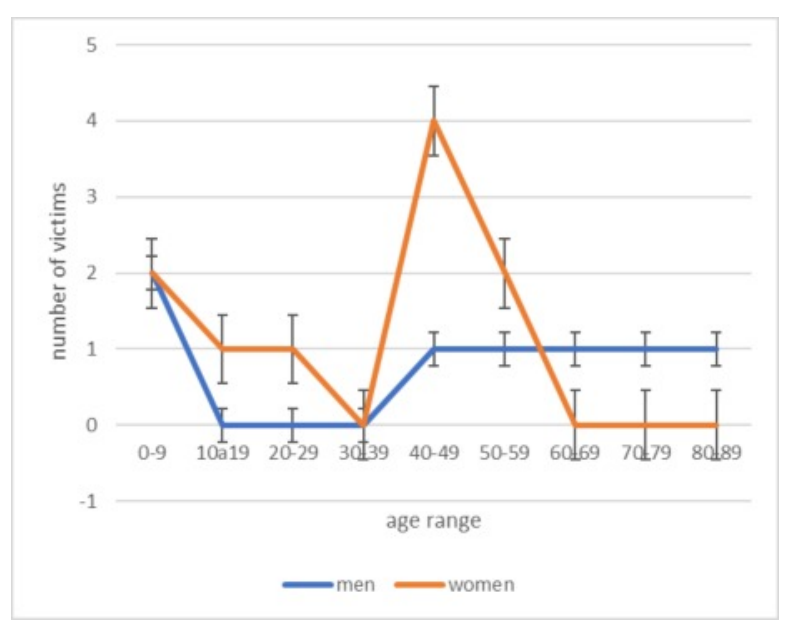

Fig. 1: Violência familiar - diferenças de faixas etárias entre homens e mulheres

A Figura 2 mostra que as mulheres sofreram violência ao longo do ciclo vital, mas as agressões por parceiros apresentaram picos na adolescência (15 - 19 anos) e juventude ( 25 - 29 anos) e a maioria de casos de violência no âmbito familiar ocorreu entre 40 e 55 anos de idade. Neste estudo, VPI e VF em mulheres apresentaram distribuição inversa conforme a faixa etária, sendo que $56,6 \%$ das mulheres vítimas de VPI (17 casos) e $23,5 \%$ das mulheres vítimas de VF (4 casos) tinham menos de 30 anos de idade. Acima de 40 anos de idade, foram observados 5 casos de VPI (16,6\% da amostra) e 6 casos de VF $(35,2 \%$ da amostra). 


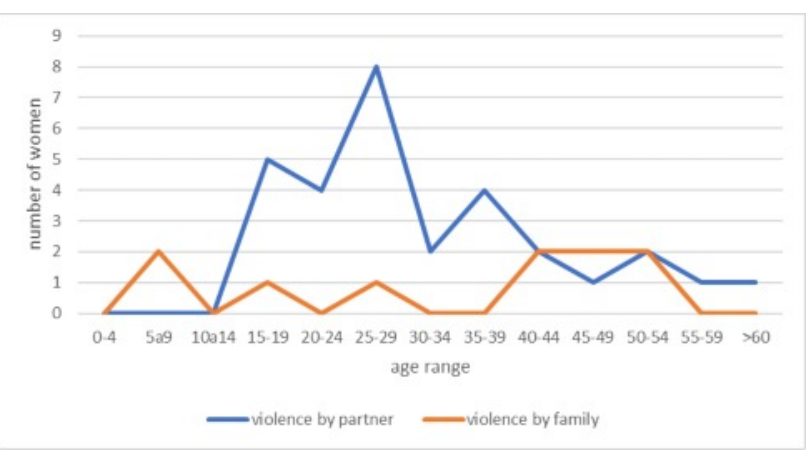

Figura 2. Faixas etárias de mulheres vítimas de violência familiar e por parceiro íntimo

\section{Procedência}

Conforme a Figura 3, a maioria das vítimas que notificaram violência perpetrada por parceiro íntimo (VPI) residiam na capital (18 casos ou 60\% da amostra). Dados semelhantes foram observados para os casos de violência familiar (VF), onde $64,7 \%$ da amostra (11 casos) também tinham domicílio na capital.

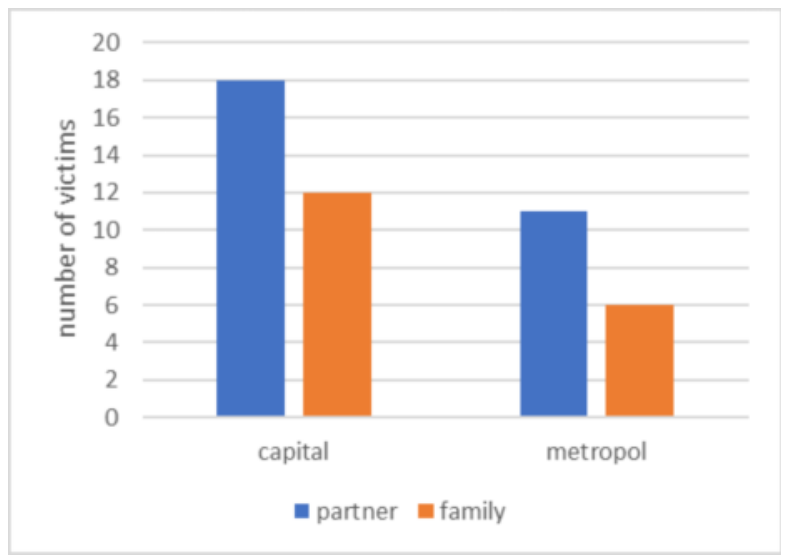

Figura 3. Procedência das vítimas de violência por parceiro íntimo e familiar

\section{Relacionamento com o agressor}

Nos casos de violência familiar (VF), todas as crianças e adolescentes foram agredidas pelas figuras de proteção - mães, pais e padrasto. Da mesma forma, todas as vítimas com mais de 60 anos de idade sofreram violência física por parte de filhos ou genros. Nas demais faixas etárias os agressores domésticos foram sobrinhos, cunhados, enteados, genros e irmão.
Na violência por parceiro íntimo (VPI), os agressores conviviam com as vítimas em 11 casos (36,6\% da amostra) - maridos/esposa, namorados ou companheiros. Todas as mulheres com menos de vinte anos residiam com os companheiros quando realizaram a notificação policial e uma estava grávida do agressor. Três vítimas (10,0\%) estavam em processo de separação e $53 \%$ da amostra (16 casos) foram agredidas por ex-companheiros, exmaridos ou ex-namorados. O tempo de separação entre a vítima e o agressor variou entre 15 dias e dois anos.

\section{Tipo de violência}

A violência doméstica familiar (VF) foi produzida predominantemente por instrumento contundente através do emprego das mãos em $94,1 \%$ da amostra (16 casos). Houve um caso de agressão sexual, em vítima adulta, portadora de necessidades especiais. A violência por parceiro íntimo foi exclusivamente por agressão física em 14 casos ou 46,6\% da amostra, produzida por golpes com as mãos, mordidas e puxões de cabelos. Uma vítima relatou agressão com faca. Duas mulheres foram resgatadas em cárcere privado, onde relataram violência física, psicológica e sexual. Uma única vítima relatou violência psicológica exclusiva praticada pelo companheiro. Em $43,3 \%$ da amostra (13 casos) houve relato de violência física e psicológica, evoluindo para ameaças de morte e stalking. O único caso masculino de VPI envolveu violência física.

\section{Vitimização prévia}

Em um terço das vítimas (33,3\% da amostra) havia notificação policial anterior de violência por parceiro íntimo (atual ou anterior), na forma de violência física e/ou psicológica.

\section{Opinião sobre o isolamento social na dinâmica da violência}

As mulheres vítimas de violência pelo parceiro responderam em $90,3 \%$ da amostra (28 casos) que a quarentena não modificou a frequência ou intensidade dos atos abusivos. Entretanto, para 3 mulheres entre 21 e 36 anos de idade $(9,6 \%$ da amostra), os fatores estressores relacionados com o isolamento social contribuíram para o aumento da violência. 


\section{Solicitação de Medida Protetiva}

No momento da notificação policial, 20 mulheres (68,9\% da amostra) vítimas de violência doméstica perpetrada pelos parceiros solicitaram medidas protetivas de urgência no momento da notificação policial, mas nenhuma aceitou proteção em abrigos. Em oito casos (27,5\% da amostra), as mulheres tinham medidas protetivas anteriores contra 0 agressor.

\section{DISCUSSÃO}

Neste estudo foi observado que houve predomínio de vítimas do sexo feminino em ambas modalidades de violência doméstica, sendo que $96,6 \%$ das mulheres sofreram violência por parceiro íntimo (VPI) e $58,8 \%$ por outros membros da família (VF). A violência doméstica contra homens foi verificada nas faixas etárias extremas: infância e terceira idade. Embora os homens também possam ser vítimas de violência doméstica, as mulheres são imensamente mais afetadas por este tipo de violência, nas diversas formas apresentadas: física, sexual, psicológica, econômica e stalking. Ao longo do ciclo vital, estima-se que $35 \%$ das mulheres terão experimentado violência física/sexual por parceiro íntimo ou familiar $(8,15)$.

As crianças e adolescentes foram vítimas de violência familiar em $29,4 \%$ da amostra e $16,6 \%$ dos casos de violência por parceiro íntimo envolveram meninas entre 16 e 19 anos de idade. Estudos sobre doenças e crises humanitárias anteriores permitem antever que a pandemia Covid-19 pode agravar os riscos pré-existentes de violência doméstica, principalmente contra meninas adolescentes (16). Com o isolamento social, muitas crianças permaneceram nas residências com os abusadores e sem o espaço de proteção que as escolas usualmente forneciam (17). No Brasil, as políticas eficientes em saúde pública reduziram as taxas de mortalidade infantil nos primeiros anos de vida. Entretanto, crianças pequenas permanecem predispostas à violência intrafamiliar, devido à sua condição de dependência dos cuidadores e de poucas interações no ambiente não doméstico $(18,19,20)$. Estima-se que, anualmente, um bilhão de crianças sofram violência física, psicológica ou sexual em torno do mundo e, em 2017, 40 mil crianças foram vítimas de homicídio. Levantamento de 70 assassinatos de meninas e adolescentes em
Porto Alegre, entre 2010 e 2016, mostrou que $72,7 \%$ das vítimas de feminicídio (parceiro íntimo) tinham entre 13 e 15 anos de idade. (20)

Poucas pesquisas nacionais abordam a violência no namoro e nas relações íntimas entre jovens. Entretanto, organizações internacionais descrevem a problemática das uniões maritais precoces e de casamentos infantis, onde a violência pode começar nas fases iniciais do relacionamento, afetando principalmente as meninas. Essa cultura de violência nas relações de namoro, o ciúme e a posse acabam gerando processos violentos de poder e subordinação $(18,20,21)$. Neste estudo, a agressão física na gestação e o cárcere privado foram descritos pelas adolescentes. Na quarentena, a presença das adolescentes gestantes em casa pode favorecer $\mathrm{o}$ abandono escolar e estimular casamentos precoces ou forçados, considerando a necessidade de cuidados com o futuro bebê e questões econômicas (16).

As mulheres jovens (menos de 30 anos) foram mais atingidas pela violência praticada por parceiros (56,6\% da amostra). Entre as mulheres que já possuíam alguma medida protetiva de urgência, $75 \%$ dos casos (seis mulheres) estavam nessa faixa etária. A notificação dos fatos em muitos casos agravou o grau de violência do agressor e algumas mulheres relataram novo episódio violento ou ameaça de morte após a procura de ajuda. Há relato de dois agressores que desafiaram autoridades e vizinhos demonstrando o sentimento de posse sobre a vítima. A literatura descreve esse olhar perverso do parceiro sobre a mulher, considerandoa como objeto e não como pessoa (15). Na quarentena, os desejos de poder, dominação e submissão podem ser potencializados e culminar com o grau máximo de violência na relação - o feminicídio (22).

A pandemia trouxe efeitos sociais significativos a partir da aproximação forçada com os membros da família. Fatores estressores, sentimentos de confusão e raiva acabam gerando a tendência a comportamentos explosivos que podem durar semanas ou meses, aumentando o risco de violência doméstica $(1,23,24,25)$. Em nosso estudo, as pessoas com mais de sessenta anos ( 3 casos - 17,6 $\%$ da amostra) foram agredidas por seus filhos ou genros. Todos os idosos pertenciam ao sexo masculino e, em duas situações, foram agredidos 
enquanto defendiam suas filhas de violência por parte de seus parceiros.

Como limitação ao estudo, observamos que a amostra correspondeu à parcela de vítimas que realizaram exame na clínica forense e, espontaneamente, solicitaram acolhimento no setor psicossocial. Desta forma, não corresponde ao número total de casos de violência doméstica atendida no período, não sendo possível fazer inferência sobre o aumento do número de casos de violência doméstica no período analisado.

\section{CONSIDERAÇÕES FINAIS}

A violência no ambiente doméstico atinge predominantemente as pessoas em situação de vulnerabilidade como as crianças, mulheres e idosos. Durante o isolamento social, crianças e idosos podem estar mais vulneráveis à violência familiar e mulheres à violência por parceiro íntimo. A presença do agressor no domicílio e a diminuição das atividades externas deixam as vítimas na dependência de encontrar um pretexto para poder se ausentar e procurar atendimento. Além das restrições financeiras e de movimentos, as mulheres acabam vivenciando o aumento do trabalho doméstico, dos cuidados com crianças, idosos e familiares doentes. Por estas razões, é importante divulgar amplamente as formas de notificação dos diversos tipos de violência no ambiente doméstico, facilitando o acesso das vítimas aos órgãos de proteção, aumentando o número de equipes destinadas ao atendimento dessa demanda e ofertando meios inovadores de pedir socorro, como o código utilizado nas farmácias da Espanha, França e Brasil.

\section{CONFLITO DE INTERESSES}

Os autores declaram não apresentar conflitos de interesses.

\section{REFERÊNCIAS BIBLIOGRÁFICAS}

1. USHER. K; BHULLAR. N; DURKIN. J; GYAMFI. N; JACKSON. D. Family violence and COVID-19: Increased vulnerability and reduced options for support. International Journal of Mental Health Nursing (2020) 29, 549-552. Acesso em 14/07/2020. Disponível em: https://www.ncbi.nlm.nih.gov/pmc /articles/PMC7264607
2. ABEL. T; MCQUEEN. D. The COVID-19 pandemic calls for spatial distancing and social closeness: not for social distancing. International Journal of Public Health (2020) 65:231

3. NICOLA. M; ALSAFI. ZAID; SOHRABI. C et al. The socio-economic of the coronavirus pandemic (COVID-19): a review. International Journal of Surgery 78 (2020) 185-193

4. TELLES. L; VALENÇA. A; BARROS. A; SILVA. A. Domestic violence in the COVID-19 pandemic: a forensic psychiatric perspective. Braz J Psychiatry. 2020;00:000-000. Access in 14 July 2020. Disponível em: http://dx.doi.org/10.1590/1516-4446-20201060

5. SACCO. $M$ et al. The impact of the Covid-19 pandemic on domestic violence: The dark side of home isolation during quarantine. Medico-Legal Journal 0(0) 1-3. 2020. DOI: 10.1177/002581722093 0553

6. NEETU. J, CASEY. S, CARINO. G, McGOVERN. T. Lessons never learned: crisis and gender-based violence. Developing World Bioeth. 2020;20:65-68. 7. THOMAS. M; RAJAN. S. Intimate partner violence (IPV) in the wake of COVID-19 in India: a conceptual overview. The International Journal of Indian Psychology. 2020;8(2).

8. WORLD HEALTH ORGANIZATION (WHO). Violence and injury prevention. Accessed 13 July 2020. Disponível em: https: www.who.int/violence_injury_prevention/violenc e/en

9. DUNCAN. E. NSW domestic violence support groups warn coronavirus isolation is prompting surge in demand for services. ABC News Australia. Accessed 21 July 2020. https://www.abc.net.au/ne ws/2020-03-27/coronavirus-domestic-familyviolence-covid-19-surge/12096988

10. BRADBURY-JONES. R; ISHAM. L. The pandemic paradox: the consequences of COVID-19 on domestic violence. J Clin Nurs. 2020;29:2047-2049. 11. CAMPBELL. A. An increasing risk of family violence during the Covid-19 pandemic: Strengthening community collaborations to save lives. Forensic Sci Int Rep. (2020). Accessed 14 July 2020. https://doi.org/10.1016/j.fsir.2020.100089

12. AGI. J. Crime rate in WA plunges amid coronavirus social distancing lockdown measures. ABC News Australia. Accessed 22 July 2020. https://www.abc.net.au/news/2020-04- 
08/coronavirus-shutdown-sees-crime-rate-drop-inwa/12132410

13. COSTA. P. Violências contra mulheres em tempos de 2020. http://www.ufs.br/conteudo/65089-violenciascontra-mulheres-em tempos-de covid-19

14. MARQUES. E; MORAES. C; HASSELMAN. M; DESLANDES. S; REICHENHEIM. M. Violence against women, children, and adolescents during the COVID-19 pandemic: overview, contributing factors, and mitigating measures. Cad. Saúde Pública 2020;36(4). Acesso 14/07/2020. Disponível em: https://www.scielosp.org/article/csp/2020.v36n4/ e00074420/

15. VIEIRA. R; GARCIA. L; MACIEL. E. Isolamento social e o aumento da violência doméstica: o que isso nos revela? Rev. bras. epidemiol., Rio de Janeiro, vol. 23, 2020. Access on 14 July 2020. Disponível em: https://www.scielo.br/scielo.php?s cript=sci_arttext\&pid=S1415-790X2020000100201 16. UNICEF. Countries failing to prevent violence against children, agencies warn. Global status report on preventing violence against children calls for more government action and warns of 'dramatic impact' of COVID-19. Access on 17 July 2020. Disponível em: https://www.unicef.org/pressreleases/countries-failing-prevent-violence-againstchildren-agencies-warn

17. UNICEF. COVID-19-GBV risks to adolescent girls and interventions to protect and empower them. International Rescue Committee. Access on 17 July 2020. Disponível em: https://www.unicef.org/docu ments/covid-19-gbv-risks-adolescent-girls-andinterventions-protect-and-empower-them

18. UNICEF. Annual Results Report 2016. Gender Equality. New York: The United Nations Children's Fund; 2017 [accessed in 2018 Apr 02]; Available from: https:www.unicef.org/publicpartnerships/file s/2016arr-gender(1).pdf

19. UNICEF. La violencia en la primera infancia. Marco regional de UNICEF para América Latina y EI Caribe. Panama: The United Nations Children's Fund; 2017 [accessed in $2018 \mathrm{Apr}$ 02]. Available from: https://www.unicef.org/lac/sites/unicef.org. lac/files/2018-03/20171023_UNICEF_LACRO_Fram eworkViolencia_ECD_ESP.pdf

20. RIOS. A; MARTINI. M; CRESPO. K; MORALES. A; MAGALHÃES. P; TELLES. L. Sociodemographic, criminal and forensic characteristics of a sample female of female children and adolescents murdered in Brazil. 2010-2016. Rev. Fac. Med. 2019;67(3)

21. Martins, AP. Violência no namoro e nas relações íntimas entre jovens: considerações preliminares sobre o problema no Brasil. Gênero. 2017;17(2):9-28. 22. MARANHÃO. R. Domestic violence during the quarantine of COVID-19: between novels, femicides and prevention. Braz. J. Hea. Rev., v. 3, n. 2, p.31973211, 2020.

23. BAVEL. J et al. Using social and behaviourial science to support COVID-19 pandemic response. Nature Human Behaviour, vol.4, 2020, 460-471. Access in 14 July 2020. Disponível em: https://www.nature.com./nathumbehav

24. BOSERUP. B; McKENNEY. M; ELKBULI. A. Alarming trends in US domestic violence during the COVID-19 pandemic. American Journal of Emergency Medicine. Accessed 13 July 2020. https://doi.org/10.1016/j.ajem.2020.04.077

25. BOUILLON-MINOIS. J; CLINCHAMPS. M; DUTHEIL. F. Coronavirus and quarantine: catalysts of domestic violence. Violence Against Women. 2020;1-3. DOI:10.1177/1077801220935194

26. GELDER. N; PETERMAN. A; POTTS. A et al. COVID-19: Reducing the risk of infection might increase the risk of intimate partner violence. EClinicalMedicine 21 (2020). Accessed 17 July 2020. https://doi.org/10.1016/j.eclinm.2020.100348

\section{CONTRIBUIÇÕES}

Angelita Maria Ferreira Machado Rios - autor principal

Alexia Oro dos Santos - revisão bibliográfica e tradução

Larissa de Oliveira Silveira - revisão bibliográfica e tradução

Laura Chies Kercher - revisão bibliográfica e tradução Letiane Montagner Ifarraguirre - revisão bibliográfica e tradução Livia Capuano Fogaça - coordenação Eduarda Pasini - revisão bibliográfica Martha Rocha - responsável pela coleta de dados Vanessa Machado Rios - revisão literatura e tradução final 